UT-11-20

\title{
Domain Walls and Gravitational Waves after Thermal Inflation
}

\author{
Takeo Moroi and Kazunori Nakayama \\ Department of Physics, \\ University of Tokyo, Bunkyo-ku, \\ Tokyo 113-0033, Japan
}

(Dated: October 30, 2018)

\begin{abstract}
Thermal inflation is an attractive solution to the cosmological moduli problem. However, domain walls may be formed after thermal inflation and some mechanisms are needed to eliminate the domain wall before it dominates the Universe. We point out that gravitational waves produced by the dynamics of domain walls may be observed by the pulsar timing experiments and future spaceborne gravitational wave detectors, which provides a probe into the period of thermal inflation. We also show that the QCD instanton effect can effectively eliminate the domain walls with producing observable amount of gravitational waves.
\end{abstract}




\section{INTRODUCTION}

While the supergravity and string theory are well-motivated candidates of the fundamental theory, there often appear light scalar fields, called moduli, having Planck-scale suppressed interactions with matter. The moduli cause serious cosmological problems, since the lifetime is typically longer than 1 sec and the energy density stored in the form of coherent oscillation is too much. The decay of moduli usually injects significant amount of energy after the big-bang nucleosynthesis $(\mathrm{BBN})$ begins, and it modifies subsequent cosmological scenarios. This is called the cosmological moduli problem [1, 2]. Even if the moduli are heavy enough to decay before BBN, gravitinos produced by the moduli decay again may be problematic [3]].

Thermal inflation [4 7] is a short period of inflation which takes place well after the primordial inflation. It is regarded as a solution to the cosmological moduli problem, since the coherent oscillation of the moduli is sufficiently diluted by the exponential expansion during thermal inflation and subsequent entropy-production by the flaton decay. Thermal inflation is driven by a scalar field, called flaton, having a flat potential and trapped at the origin of the scalar potential due to thermal effects. This kind of scalar field can be embedded into supersymmetric (SUSY) theories.

However, the most thermal inflation models suffer from a problematic domain wall (DW) formation after the phase transition in association with the end of thermal inflation [7]. This is because the flaton usually has $Z_{n}$ symmetry which is spontaneously broken at the true vacuum. Once formed, DWs eventually dominate the energy density of the Universe resulting in a cosmological disaster. Thus the $Z_{n}$ symmetry, which guarantees the flatness of the flaton potential, must be explicitly broken to some extent in order for the DWs to disappear before they come to dominate the Universe. Actually, an introduction of a small

explicit breaking in the scalar potential can solve the DW problem [8, 9]. Such DWs can be a source of gravitational waves (GWs).

In this paper, we study the GWs from the dynamics of DWs produced after thermal inflation. We show that the amount of the GWs may be within the reach of the pulsar timing experiments and future space-borne GW detectors. Thus the study of GWs provides a way to probe the period of thermal inflation. It is also shown that the $Z_{n}$ can be naturally broken by the QCD instanton effect in a simple class of thermal inflation model; in such a 
model, the abundance of the GWs will be well within the observable range.

\section{THERMAL INFLATION MODEL}

Let us start with the model of thermal inflation. We introduce a gauge singlet superfield $\phi$ which takes the role of the flaton. ${ }^{1}$ Imposing $Z_{n}$ symmetry, under which $\phi$ transforms as $\phi \rightarrow e^{2 \pi i / n} \phi$, we adopt the following superpotential ${ }^{2}$

$$
W=\frac{\phi^{n}}{n M^{n-3}}+k \phi Q \bar{Q}+W_{0}
$$

Here, $Q$ and $\bar{Q}$ are additional "quarks" which have quantum numbers for $\mathrm{SU}(3)_{\mathrm{QCD}}$; they are thermalized when $\phi=0$, and give a thermal mass to $\phi$. In addition, $W_{0}$ is a constant which is related to the gravitino mass $m_{3 / 2}$ as $W_{0}=m_{3 / 2} M_{P}^{2}$ (with $M_{P}$ being the reduced Planck scale). In the following, in order to guarantee the flatness of the potential of $\phi$, we take $n \geq 4$. We also comment here that, if $W_{0}=0$, the superpotential has an $R$-symmetry under which $\phi$ has a charge $+2 / n$. Although such an $R$-symmetry is explicitly broken by the constant term $W_{0}$, we will be interested in the case that the effect of the breaking is relatively small. Then, in the vacuum where $\langle\phi\rangle \neq 0$, quasi Nambu-Goldstone boson, which we call $R$-axion, shows up.

Including the SUSY breaking effect, the zero temperature potential of the flaton $\phi$ takes the following form :

$$
V=V_{0}-m^{2}|\phi|^{2}+(n-3)\left(\frac{A \phi^{n}}{n M^{n-3}}+\text { h.c. }\right)+\frac{|\phi|^{2(n-1)}}{M^{2(n-3)}} .
$$

If there are no sources for the $A$-parameter other than $W_{0}$, we have $A=m_{3 / 2}$. The SUSY breaking mass parameter for $\phi$ is represented by $-m^{2}$ and is assumed to be negative. Hereafter we take $A$ real and positive. In addition, we take $A \ll m$ for simplicity. The vacuum expectation value (VEV) of $\phi$ is given by

$$
\langle\phi\rangle \equiv v=\left(\frac{m M^{n-3}}{\sqrt{n-1}}\right)^{1 /(n-2)} .
$$

\footnotetext{
1 We use a same symbol for a superfield as its scalar component.

2 Terms like $\phi^{2 n}, \phi^{3 n}, \ldots$ are also allowed, but they have little effects on the flaton dynamics discussed below and hence are neglected.
} 
The flaton mass around the minimum is given by $m_{\phi}=\sqrt{2(n-2)} m$, and $V_{0}$ is determined as

$$
V_{0}=\frac{n-2}{n-1} m^{2} v^{2}
$$

so that the cosmological constant vanishes at the vacuum. In the gravity-mediated SUSY breaking model, $m$ is expected to be of the order of TeV. In the gauge-mediated SUSY breaking model [10], on the other hand, the scalar potential is slightly more complicated [11, 12] and the parameter dependence changes. Hereafter, we treat $v$ and $m_{\phi}$ as free parameters without specifying the mechanism of SUSY breaking.

Suppose that $\phi$ is trapped at the origin due to the large Hubble mass term during/after inflation. In such a period, $Q$ and $\bar{Q}$ are massless and hence they participate to thermal bath. Thus the flaton obtains a thermal mass of $m_{T}^{2} \sim k^{2} T^{2}$. As the cosmic temperature decreases, the energy density of the Universe is dominated by the potential energy of $\phi$ and thermal inflation begins. Thermal inflation ends at the temperature $T_{\text {end }} \sim m$ for $k \sim \mathcal{O}(1)$. Then the flaton oscillates around the minimum, and finally it decays into radiation. We parametrize the flaton decay rate as

$$
\Gamma=\frac{c}{4 \pi} \frac{m_{\phi}^{3}}{v^{2}}
$$

with a numerical constant $c$, which depends on the decay mode. If $\phi$ decays into the Higgsino pair via the superpotential of $k^{\prime} \phi H_{u} H_{d}$ (where $H_{u}$ and $H_{d}$ are the up- and down-type Higgses, respectively) with $k^{\prime} \sim m_{\phi} / v$, then $c \sim \mathcal{O}(1)$. On the contrary, $c \sim \mathcal{O}\left(10^{-4}\right)$ for the decay into gluons via the loop effect. The reheating temperature after the flaton decay is estimated $\mathrm{as}^{3}$

$$
T_{\mathrm{R}}=\left(\frac{90}{\pi^{2} g_{*}}\right)^{1 / 4} \sqrt{\Gamma M_{P}} \simeq 6.2 \times 10^{2} \mathrm{GeV} \sqrt{c}\left[\frac{228.75}{g_{*}\left(T_{\mathrm{R}}\right)}\right]^{1 / 4}\left(\frac{m_{\phi}}{1 \mathrm{TeV}}\right)^{3 / 2}\left(\frac{10^{10} \mathrm{GeV}}{v}\right),
$$

where $g_{*}(T)$ is the effective number of massless degrees of freedom at the temperature $T$. Notice that $T_{\mathrm{R}}$ is required to be higher than a few MeV for successful BBN.

Let us estimate the modulus abundance in the presence of thermal inflation. We denote the modulus field by $\chi$ and its mass and energy density by $m_{\chi}$ and $\rho_{\chi}$. Thermal inflation

3 The flaton can also decay into the $R$-axion pair. If the decay into the $R$-axion is dominant, the final reheating completes by the decay of $R$-axion. This situation is effectively incorporated in the parametrization (5) by choosing the value of $c$ appropriately. In addition, the flatino, the fermionic component of the flaton, has a mass of $\sqrt{n-1} \mathrm{~m}$, and hence the flaton cannot decay into the flatino pair. 
efficiently dilutes the moduli through the late-time entropy production. The dilution factor is defined as the ratio of the entropy with and without the additional entropy production at the flaton decay. It is given by [7, 8].

$$
\Delta=\frac{30}{\pi^{2} g_{*}\left(T_{\text {end }}\right)} \frac{V_{0}}{T_{\text {end }}^{3} T_{\mathrm{R}}} \simeq 1.3 \times 10^{15}\left[\frac{228.75}{g_{*}\left(T_{\text {end }}\right)}\right]\left(\frac{1 \mathrm{TeV}}{m}\right)\left(\frac{1 \mathrm{GeV}}{T_{\mathrm{R}}}\right)\left(\frac{v}{10^{10} \mathrm{GeV}}\right)^{2} .
$$

The modulus abundance after the entropy production is

$$
\begin{aligned}
\left(\frac{\rho_{\chi}}{s}\right)^{(\text {prim })} & =\frac{1}{\Delta} \times \frac{1}{8} T_{\mathrm{R}}^{(\mathrm{inf})}\left(\frac{\chi_{0}}{M_{P}}\right)^{2} \\
& \simeq 1 \times 10^{-7} \mathrm{GeV} \sqrt{c}\left(\frac{m}{1 \mathrm{TeV}}\right)^{5 / 2}\left(\frac{10^{10} \mathrm{GeV}}{v}\right)^{3}\left(\frac{T_{\mathrm{R}}^{(\mathrm{inf})}}{10^{6} \mathrm{GeV}}\right)\left(\frac{\chi_{0}}{M_{P}}\right)^{2}
\end{aligned}
$$

where $\chi_{0}$ is the initial amplitude of the modulus. Here we have assumed that the modulus begins to oscillate before the inflaton decays and also that the inflaton decays before thermal inflation starts. Otherwise, the expression becomes more complicated [8]. We call this "primary" moduli for the reason discussed below.

There is another contribution to the modulus oscillation [7]. Since the potential minimum of the moduli during thermal inflation may be displaced from the true minimum due to the Hubble mass correction, the secondary oscillation is induced after thermal inflation ends. The amplitude is estimated as $\delta \chi \sim V_{0} \chi_{*} /\left(m_{\chi}^{2} M_{P}^{2}\right)$. Hence the moduli abundance is estimated to be

$$
\begin{aligned}
\left(\frac{\rho_{\chi}}{s}\right)^{(\mathrm{sec})} & =\frac{m_{\chi}^{2}(\delta \chi)^{2} / 2}{V_{0}} \frac{3 T_{\mathrm{R}}}{4} \\
& \simeq 1 \times 10^{-15} \mathrm{GeV} \sqrt{c}\left(\frac{m}{1 \mathrm{TeV}}\right)^{7 / 2}\left(\frac{1 \mathrm{TeV}}{m_{\chi}}\right)^{2}\left(\frac{v}{10^{10} \mathrm{GeV}}\right)\left(\frac{\chi_{*}}{M_{P}}\right)^{2}
\end{aligned}
$$

Here $\chi_{*}$ is the true minimum of the modulus while the origin is chosen so that the Hubble mass correction is given by $H^{2} \chi^{2}$. This is called the "secondary" moduli. The final modulus abundance is the sum of the primary and secondary moduli. Which one dominates the moduli density depends on various parameters. BBN, cosmic microwave background, and diffuse $X$ - and $\gamma$-ray background give stringent upper bound on the modulus abundance, depending on its mass. Complete analyses on the modulus abundance and its cosmological effects can be found in Ref. [8]. 


\section{DOMAIN WALL PROBLEM AND A SOLUTION}

In this section we discuss the problematic DW formation after thermal inflation, and a solution to the DW problem.

After the decay, the flaton randomly falls into one of the $n$-degenerate minima. Since the VEV of $\phi$ spontaneously breaks the $Z_{n}$-symmetry, DWs are formed after thermal inflation. The DW tension is estimated as

$$
\sigma \simeq \frac{V_{A}}{m_{a}}
$$

where

$$
V_{A}=2(n-3) A \frac{v^{n}}{n M^{n-3}},
$$

and

$$
m_{a}^{2}=\frac{n(n-3)}{\sqrt{n-1}} A m
$$

gives the $R$-axion mass. DWs obey the scaling low in which about one DW exists per Hubble horizon as far as the viscosity on the DW is negligible [13]. ${ }^{4}$ Then DWs begin to dominate the Universe at

$$
H_{\mathrm{dom}} \simeq \frac{\sigma}{M_{P}^{2}}
$$

This is problematic unless $\sigma \lesssim(1 \mathrm{MeV})^{3}$, since otherwise DWs dominate the Universe before the present epoch [14]. Thus there should be an explicit $Z_{n}$ breaking term which makes DWs unstable.

Let us denote the $Z_{n}$-breaking scalar potential by $V_{\epsilon}$, whose possible origins will be discussed later. It generates a bias for the $n$ minima of the original potential and the degeneracy among those minima are lifted completely. DWs are not absolutely stable under the existence of the bias, and eventually collapse when the bias energy density becomes comparable to the DW energy density [15]. This happens when

$$
H_{\mathrm{dec}} \simeq \frac{V_{\epsilon}}{\sigma}
$$

Therefore, in order for the DWs to collapse before they come to dominate $\left(H_{\text {dec }} \gg H_{\text {dom }}\right)$, we need the following condition,

$$
V_{\epsilon} \gg \frac{\sigma^{2}}{M_{P}^{2}} .
$$

4 Actually interactions of the DW with $Q$ and $\bar{Q}$ cause friction, but this effect is found to be insignificant for the following analysis. 
The DW problem is solved if this condition is satisfied. Now we describe possible origins of the bias term.

\section{A. Small explicit $Z_{n}$ breaking term}

Let us introduce the following superpotential

$$
\delta W=\epsilon \frac{\phi^{\ell}}{\ell M^{\ell-3}},
$$

in addition to (11), where $n$ and $\ell$ are relatively prime numbers and $\epsilon$ is a small coefficient. In order for this term not to change the flaton potential significantly, we require

$$
\epsilon \frac{v^{\ell}}{\ell M^{\ell-3}} \ll \frac{v^{n}}{n M^{n-3}} \quad \leftrightarrow \quad \epsilon \ll \frac{\ell}{n}\left(\frac{v}{M}\right)^{n-\ell} .
$$

Then the following additional scalar potentials are generated,

$$
V_{\epsilon}=\epsilon A(\ell-3) \frac{\phi^{\ell}}{\ell M^{\ell-3}}+\epsilon \frac{\phi^{n-1} \phi^{* \ell-1}}{M^{n+\ell-6}}+\text { h.c.. }
$$

The second term dominates for $m>A$ and this yields the bias for the $n$ minima of the original potential as

$$
V_{\epsilon} \simeq 2 \epsilon \frac{v^{n-\ell-2}}{M^{n+\ell-6}}
$$

The original $Z_{n}$ symmetry is explicitly violated by the bias, and hence the degeneracy of the $n$ minima of the scalar potential is broken. The condition (15) is rewritten as

$$
\epsilon \gg \frac{A}{m} \frac{v^{2}}{M_{P}^{2}}\left(\frac{v}{M}\right)^{n-\ell} .
$$

If this condition is satisfied, DWs collapse before they come to dominate the Universe and there is no DW problem.

\section{B. QCD instanton effect}

Rather simple scenario to break the $Z_{n}$ symmetry is to use the QCD instanton effects [17]. This is economical in the sense that the term $\phi Q \bar{Q}$ in (1), which is needed for giving rise to a thermal mass for the flaton field, also works as a source of the bias for eliminating the DWs. ${ }^{5}$

5 This solution may be incompatible with the Peccei-Quinn mechanism [16] for solving the strong CP problem [17, 18]. In that case, we may arrange the model so that the heavy quarks $Q(\bar{Q})$ have a charge of hidden $\mathrm{QCD}$ and its scale is around $\mathrm{GeV}-\mathrm{TeV}$. 
The $Z_{n}$ symmetry in (11) has an anomaly for $\mathrm{SU}(3)_{\mathrm{QCD}}$. Consequently, the QCD instanton effect works as a bias, which lifts the classical degeneracy of the $n$ vacua. The bias due to this effect is estimated to be [19]

$$
V_{\epsilon} \simeq f_{\pi}^{2} m_{\pi}^{2} \frac{m_{u} m_{d}}{\left(m_{u}+m_{d}\right)^{2}}
$$

for temperature below $\Lambda_{\mathrm{QCD}} \sim 200 \mathrm{MeV}$, where $f_{\pi}$ and $m_{\pi}$ are the pion decay constant and mass, and $m_{u}\left(m_{d}\right)$ is the current mass of the up (down) quark. At higher temperature, this effect is suppressed by high powers of $T$ [20], and hence the bias due to the QCD instanton effect is only turned on at $T \sim \Lambda_{\mathrm{QCD}}[17,18,21,22]$.

We need two conditions for this mechanism to work successfully. One is Eq. (15) for DWs collapse due to the bias. The other is $H_{\mathrm{dec}} \lesssim \Lambda_{\mathrm{QCD}}^{4} /\left(T_{\mathrm{R}}^{2} M_{P}\right)$, since otherwise the QCD instanton effect is highly suppressed and it is not suitable for the bias. ${ }^{6}$ These two conditions are written as

$$
T_{\mathrm{R}}^{2} M_{P}<\sigma<\Lambda_{\mathrm{QCD}}^{2} M_{P}
$$

for $T_{\mathrm{R}}<\Lambda_{\mathrm{QCD}}$. Therefore, for example, $M \sim 10^{15} \mathrm{GeV}$ and $A=m_{3 / 2} \sim m \sim 1 \mathrm{GeV}$ are good choices for $n=4$. In this choice we have $H_{\mathrm{dec}} / H_{\mathrm{dom}} \sim \mathcal{O}(1)$. As will be seen, this predicts observable GWs.

It should be noticed that $M$ cannot take an arbitrary value in order to solve the cosmological moduli problem. As seen in Eqs. (8) and (9), the moduli abundance crucially depends on $v$, which is determined by the cutoff scale $M$. Comparing with the BBN bound $\rho_{\chi} / s \lesssim 10^{-14} \mathrm{GeV}$ for the moduli of $10 \mathrm{GeV} \lesssim m_{\chi} \lesssim 1 \mathrm{TeV}[23], M \gtrsim 10^{15} \mathrm{GeV}$ for $n=4$ may be allowed for moderate parameter choices.

\section{GRAVITATIONAL WAVES FROM COLLAPSING DOMAIN WALLS}

Now we are at the position to discuss the main subject of this paper, which is the GW production from DWs. We will show that the GWs from the DWs after thermal inflation may be observable by future experiments.

The DW network has complicated structure. Large amount of energy is localized around DWs, which becomes the source GWs. As a result, considerable amount of GWs can be

6 The latter condition is based on the assumption that the Universe is flaton dominated at the QCD phase transition. If the Universe is radiation dominant at the QCD phase transition, the condition is severer. 
emitted by the relativistic motion of DWs. Frequencies of the GWs correspond to typical scales of the DW motion, which ranges from the Hubble scale to the size of DW width.

Detailed calculations of the GW spectrum from DWs using three dimensional lattice simulation were performed in Refs. [24 26]. According to recent studies [25, 26], GWs have rather broad spectrum which extends from the horizon size at the DW collapse for lower frequency side to the DW width for the higher frequency side. The GW spectrum, in terms of $\Omega_{\mathrm{GW}}(f) \equiv\left(d \rho_{\mathrm{GW}} / d \ln f\right) / \rho_{c 0}$ (where $\rho_{\mathrm{GW}}(f)$ is the GW energy density with frequency $f$ measured at present and $\rho_{c 0}$ is the present critical energy density) is almost flat between these frequencies. The energy density of gravitational waves at the collapse of DWs can be estimated as [27]

$$
\rho_{\mathrm{GW}}\left(H_{\mathrm{dec}}\right) \simeq G_{N} \frac{M_{\mathrm{DW}}^{2}}{H_{\mathrm{dec}}^{-1}} \frac{1}{H_{\mathrm{dec}}^{-3}} \sim \frac{\sigma^{2}}{M_{P}^{2}},
$$

where $G_{N}$ is the Newton constant. Here we have substituted $M_{\mathrm{DW}}=\sigma H_{\mathrm{dec}}^{-2}$. Then, $\Omega_{\mathrm{GW}}$ is given by

$$
\begin{array}{r}
\Omega_{\mathrm{GW}}(f) \simeq \Omega_{\mathrm{r}}\left[\frac{g_{*}\left(T_{\mathrm{dec}}\right)}{g_{* 0}}\right]\left[\frac{g_{* s 0}}{g_{* s}\left(T_{\mathrm{dec}}\right)}\right]^{4 / 3}\left(\frac{H_{\mathrm{dom}}}{H_{\mathrm{dec}}}\right)^{2} \text { for } H_{\mathrm{R}}>H_{\mathrm{dec}} \\
\Omega_{\mathrm{GW}}(f) \simeq \Omega_{\mathrm{r}}\left[\frac{g_{*}\left(T_{\mathrm{dec}}\right)}{g_{* 0}}\right]\left[\frac{g_{* s 0}}{g_{* s}\left(T_{\mathrm{dec}}\right)}\right]^{4 / 3}\left(\frac{H_{\mathrm{dom}}}{H_{\mathrm{dec}}}\right)^{2}\left(\frac{H_{\mathrm{R}}}{H_{\mathrm{dec}}}\right)^{2 / 3} \text { for } H_{\mathrm{R}}<H_{\mathrm{dec}}
\end{array}
$$

for $f_{\text {edge }}<f<f_{\text {peak }}$ defined below, where $\Omega_{\mathrm{r}}=8.5 \times 10^{-5}$ is the present radiation energy with three massless neutrino species density. Numerically we obtain

$$
\begin{array}{r}
\Omega_{\mathrm{GW}}(f) \simeq 2 \times 10^{-5}\left[\frac{228.75}{g_{*}\left(T_{\mathrm{dec}}\right)}\right]^{1 / 3}\left(\frac{H_{\mathrm{dom}}}{H_{\mathrm{dec}}}\right)^{2} \text { for } H_{\mathrm{R}}>H_{\mathrm{dec}}, \\
\Omega_{\mathrm{GW}}(f) \simeq 2 \times 10^{-5}\left[\frac{228.75}{g_{*}\left(T_{\mathrm{dec}}\right)}\right]^{1 / 3}\left(\frac{H_{\mathrm{dom}}}{H_{\mathrm{dec}}}\right)^{2}\left(\frac{H_{\mathrm{R}}}{H_{\mathrm{dec}}}\right)^{2 / 3} \text { for } H_{\mathrm{R}}<H_{\mathrm{dec}},
\end{array}
$$

for $f_{\text {edge }}<f<f_{\text {peak }}$. The typical frequency at the lower side, corresponding to the horizon size at the DW collapse, called the "edge" frequency $\left(f_{\text {edge }}\right)$ in Ref. [25], is given by

$$
f_{\text {edge }}=\frac{H_{\mathrm{dec}}}{2 \pi} \frac{a\left(H_{\mathrm{dec}}\right)}{a_{0}}
$$

which is estimated as

$$
\begin{array}{r}
f_{\text {edge }} \simeq 3 \times 10^{-5} \mathrm{~Hz}\left[\frac{g_{*}\left(T_{\mathrm{dec}}\right)}{228.75}\right]^{1 / 6}\left(\frac{T_{\mathrm{dec}}}{1 \mathrm{TeV}}\right) \quad \text { for } H_{\mathrm{R}}>H_{\mathrm{dec}}, \\
f_{\text {edge }} \simeq 3 \times 10^{-5} \mathrm{~Hz}\left[\frac{g_{*}\left(T_{\mathrm{R}}\right)}{228.75}\right]^{1 / 6}\left(\frac{T_{\mathrm{R}}}{1 \mathrm{TeV}}\right)\left(\frac{H_{\mathrm{dec}}}{H_{\mathrm{R}}}\right)^{1 / 3} \text { for } H_{\mathrm{R}}<H_{\mathrm{dec}},
\end{array}
$$


where $T_{\text {dec }}$ is the temperature at which the DWs collapse. On the other hand, the "peak" frequency $\left(f_{\text {peak }}\right)$ [25], corresponding to the DW width at the collapse redshifted to the present time, is given by

$$
f_{\text {peak }}=\frac{w^{-1}}{2 \pi} \frac{a\left(H_{\mathrm{dec}}\right)}{a_{0}},
$$

where $w \simeq m_{a}^{-1}$ is the DW width. This is estimated as

$$
\begin{array}{r}
f_{\text {peak }} \simeq 1 \times 10^{10} \mathrm{~Hz}\left[\frac{228.75}{g_{*}\left(T_{\mathrm{dec}}\right)}\right]^{1 / 3}\left(\frac{m_{a}}{1 \mathrm{TeV}}\right)\left(\frac{1 \mathrm{TeV}}{T_{\mathrm{dec}}}\right) \quad \text { for } H_{\mathrm{R}}>H_{\mathrm{dec}} \\
f_{\text {peak }} \simeq 1 \times 10^{10} \mathrm{~Hz}\left[\frac{228.75}{g_{*}\left(T_{\mathrm{R}}\right)}\right]^{1 / 3}\left(\frac{m_{a}}{1 \mathrm{TeV}}\right)\left(\frac{1 \mathrm{TeV}}{T_{\mathrm{R}}}\right)\left(\frac{H_{\mathrm{R}}}{H_{\mathrm{dec}}}\right)^{2 / 3} \text { for } H_{\mathrm{R}}<H_{\mathrm{dec}} .
\end{array}
$$

We approximate that the GW spectrum is flat between $f_{\text {edge }}$ and $f_{\text {peak }}$, although the simulation shows a bit preference for the blue spectrum [25]. In that sense, our estimate of the $\mathrm{GW}$ is conservative. It may be within the observable range of GW detectors such as pulsar timing array or space laser interferometers [28].

Fig. [1 shows the edge frequency $f_{\text {edge }}$ (left) and $\Omega_{\mathrm{GW}}$ (right) as a function of $H_{\mathrm{dec}} / H_{\mathrm{dom}}$, which parameterizes the epoch of the collapse of DWs. Here we have taken $m=1 \mathrm{TeV}$, $A=100 \mathrm{GeV}, c=1, M=M_{P}$ and $n=4,5,6$. It is clear that the GW energy density becomes larger for DWs that collapse closer to the epoch of DW domination.

Fig. 2 shows contours of $\Omega_{\mathrm{GW}}, f_{\text {edge }}$ and $T_{\mathrm{R}}$ for $n=4$ (top) and $n=5$ (bottom). In the gray region denoted by "no DW", the bias is so large, i.e. $H_{\text {dec }}>\sqrt{V_{0}} / M_{P}$, that no DW formation is expected. The current limits from pulsar timing experiments [29 31] and the LIGO experiment [32] do not give stringent constraints on the model. In the same figure, we also show the prospects of the discovery reaches of up-comming projects. Projects such as SKA [33] will probe some parameter regions. Ground based GW detectors such as the advanced LIGO [34] and LCGT[35] are also sensitive to the higher frequency GWs of $f \gtrsim 100 \mathrm{~Hz}$. Here a correlation analysis of 1 year is assumed. Space based GW detector such as LISA [36] will also be sensitive to the GWs from DW collapse for wider parameter regions. Sensitivities are found in Ref. [37]. DECIGO [38] may cover the whole parameter region with $\Omega_{\mathrm{GW}} \gtrsim 10^{-18}$. The scenario with the QCD instanton as a bias term corresponds to the lower-left edge of Fig. 2 and hence future pulsar timing and/or space-borne GW experiments will be able to detect GWs. 

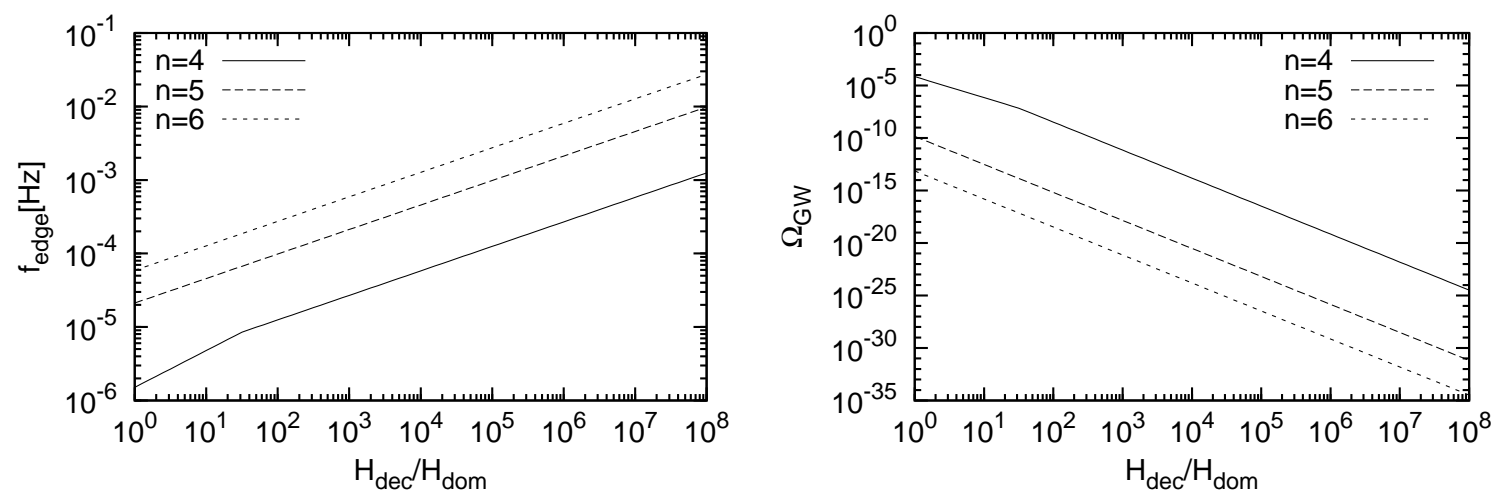

FIG. 1: The typical GW frequency $f_{\text {edge }}$ (left) and $\Omega_{\mathrm{GW}}$ (right) as a function of $H_{\mathrm{dec}} / H_{\mathrm{dom}}$. We have taken $m=1 \mathrm{TeV}, A=100 \mathrm{GeV}, c=1, M=M_{P}$ and $n=4,5,6$.

\section{DISCUSSION AND CONCLUSIONS}

Some comments are in order. It was proposed that the first order phase transition triggers the end of thermal inflation, and correspondingly bubbles of true vacuum are created [39]. Bubbles expand into the surrounding false vacuum regions and collide with each other, generating GWs with observable level [39]. This predicts higher frequency GWs than studied in this paper and hence will provide further information on the thermal inflation model.

We also comment on possible origin of the baryon asymmetry in the model. Since thermal inflation dilutes away the pre-existing baryon asymmetry, as well as the moduli, we need some mechanisms to create baryon asymmetry after thermal inflation. In this regard, a variant type of the Affleck-Dine mechanism [40] works after thermal inflation [41 45].

One might notice that the heavy quraks $Q$ and $\bar{Q}$ may be stable and cosmologically relevant [46]. Since they are once in thermal equilibrium during thermal inflation, its abundance just after the flaton decay is given by

$$
Y_{Q} \equiv \frac{n_{Q}}{s} \sim \frac{T_{\mathrm{end}}^{3} T_{\mathrm{R}}}{m^{2} v^{2}} \sim 10^{-17}\left(\frac{m_{\phi}}{1 \mathrm{TeV}}\right)\left(\frac{T_{\mathrm{R}}}{1 \mathrm{GeV}}\right)\left(\frac{10^{10} \mathrm{GeV}}{v}\right)^{2}
$$

Even if it is less than the DM abundance, BBN constraints on the strongly-interacting relic particles are much more stringent [47, 48]. ${ }^{7}$ This constraint can easily be evaded if $Q$ and $\bar{Q}$

\footnotetext{
7 The enhancement of the annihilation cross section due to the $R$-hadron formation [49] may help the situation if the flaton decays after the QCD phase transition.
} 

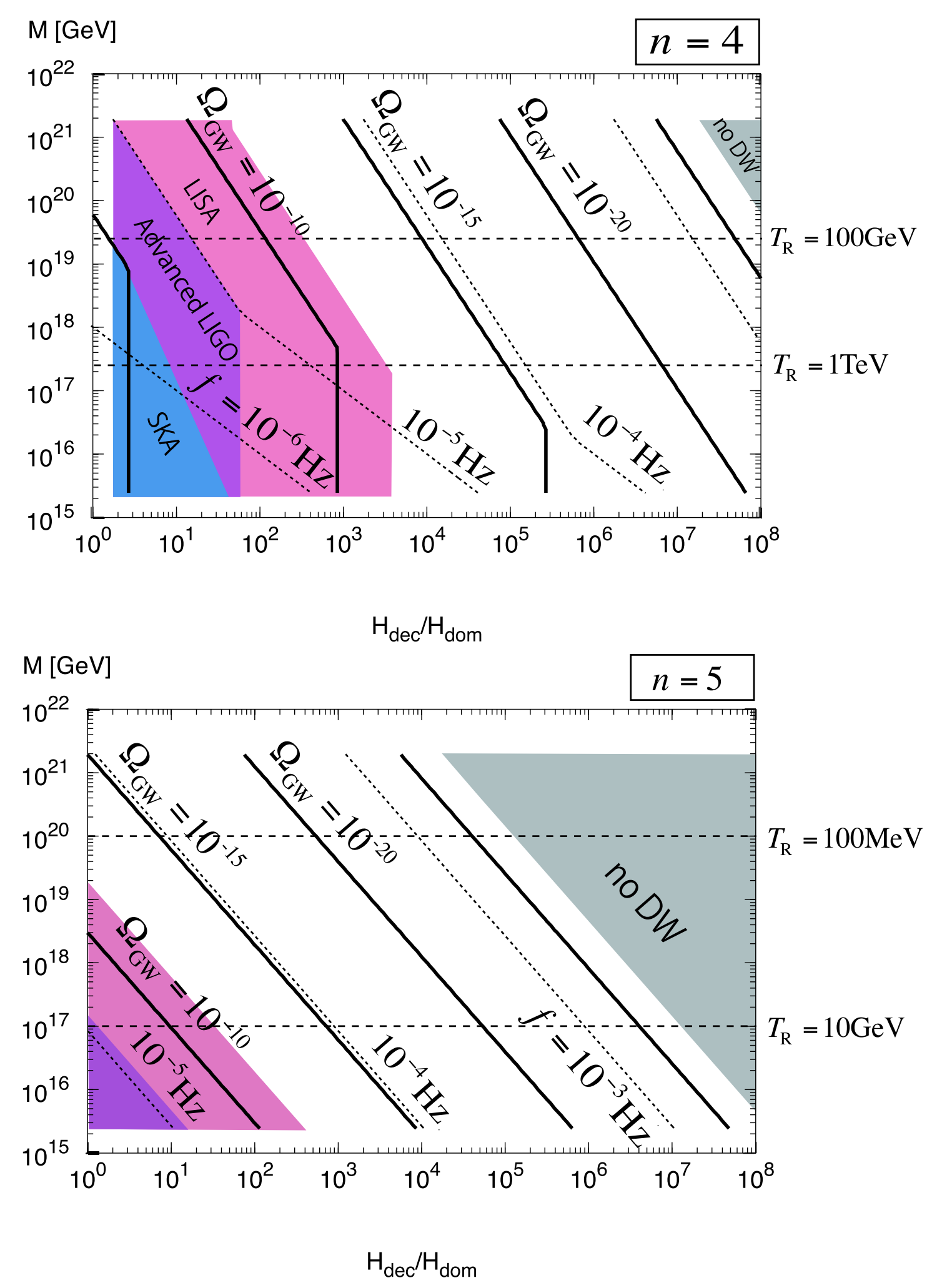

FIG. 2: Contours of $\Omega_{\mathrm{GW}}$ (solid line), $f_{\text {edge }}$ (dotted line) and $T_{\mathrm{R}}$ (dashed line) for $n=4$ (top) and $n=5$ (bottom). The regions explored by LISA, SKA and advanced LIGO are also shown. DECIGO may cover the whole parameter region with $\Omega_{\mathrm{GW}} \gtrsim 10^{-18}$. Here we have taken $m=1 \mathrm{TeV}$, $A=100 \mathrm{GeV}$ and $c=1$. 
are in complete multiplets of the GUT group, which is natural from the viewpoint of gauge coupling unification. If $Q$ and $\bar{Q}$ are embedded into fundamental and anti-fundamental representation of $\mathrm{SU}(5)_{\mathrm{GUT}}$, for example, they should be acompanied by non-colored components (denoted by $L$ and $\bar{L}$ ). The renormalization group effect makes the colored components heavier than non-colored ones at the low energy. Then, $Q$ and $\bar{Q}$ decay into $L$ and $\bar{L}$ via dimension five operators suppressed by the GUT scale. The decay rate is estimated to be $\Gamma \sim m_{Q}^{3} / M_{\mathrm{GUT}}^{2}$, where $M_{\mathrm{GUT}} \sim 10^{16} \mathrm{GeV}$ is the GUT scale. The decay rate is large enough so that colored components decay before BBN. Similarly, $L$ splits into the electrically charged and neutral components, where the former is heavier and decays into the latter. Notice that the neutral component may be a candidate of superheavy DM. ${ }^{8}$

Let us consider how robust the DW formation after thermal inflation is. In the simplest model, the flatness of the flaton potential is ensured by a $Z_{n}$ symmetry as in (11). In this case DWs necessarily appear. On the other hand, one can impose a global U(1) symmetry to make the flaton potential flat. The flaton potential is stabilized by introducing another singlet scalar with some $\mathrm{U}(1)$ charge or by taking account of the radiative correction to the flaton soft mass [45, 51 55]. Then, the spontaneous breaking of the U(1) symmetry due to the VEV of the flaton results in the formation of cosmic strings instead of DWs. Even so, the global U(1) symmetry may be anomalous and may not be a good symmetry at the quantum level. If only a discrete subgroup $Z_{N}$ of the $\mathrm{U}(1)$ symmetry remains at the quantum level, DWs still exist [56]. In particular, if $N \geq 2$, the DW problem still exists. On the contrary, if $N=1$, DWs are bounded by strings and they disappear just after the formation [57 61].

It is also possible that the $\mathrm{U}(1)$, which guarantees the flatness of the potential, is a gauge symmetry [6]. The flaton takes the role of the Higgs field that gives a mass for the U(1) gauge boson. Then, the U(1) should be an exact symmetry and DW does not exist. Instead, cosmic strings are formed which are less harmful than DWs. Cosmic strings formed after thermal inflation has a width of the $\mathrm{TeV}$ scale, while the tension can be much larger. This kind of thick strings have characteristic cosmological implications as studied in Refs. [62, 63].

Finally, we comment on another possible source of GWs, which is the DW related to the breaking of Peccei-Quinn symmetry [16]. In order to avoid the DW problem, it is often the

\footnotetext{
8 The constraints from direct detection experiments can be avoided if the mass of the neutral component is heavier than $\sim 10^{5} \mathrm{GeV}[50]$.
} 
case that axion models with $N=1$ is considered. In such a case, DWs are surrounded by cosmic strings as we have mentioned; then, DWs disappear just after the production. This happens when $T \sim \Lambda_{\mathrm{QCD}}$, so we expect that the GWs with the frequency of $f \sim 10^{-8} \mathrm{~Hz}$ are emitted from the dynamics of axionic DWs. We have estimated the amount of such GWs, and found that $\Omega_{\mathrm{GW}}$ is a few order of magnitude smaller than the sensitivity of the future pulsar timing experiments.

To summarize, a class of thermal inflation models is inevitably associated with DW formation, which reintroduces a cosmological disaster. Thus we need a mechanism to make DWs unstable. If the DW energy density at the epoch of its collapse is large enough, GWs produced by the DW collapse may be observable in future experiments. We have pointed out that such a mechanism is naturally built-in in the simplest model where the flaton couples to extra quarks as in (11); in such a model, the $Z_{n}$ symmetry has an anomaly for $\mathrm{SU}(3)_{\mathrm{QCD}}$ and the QCD instanton effects serve as a bias for lifting the degeneracy among $n$ minima. In this case the GW amplitude becomes so large that it can be well within the reach of future experiments.

\section{Acknowledgments}

K.N. would like to thank M. Kawasaki and F. Takahashi for useful conversations. This work is supported by Grant-in-Aid for Scientific research from the Ministry of Education, Science, Sports, and Culture (MEXT), Japan, No. 22540263 (T.M.), No. 22244021 (T.M.), No. 21111006 (K.N.), and No. 22244030 (K.N.).

[1] G. D. Coughlan, W. Fischler, E. W. Kolb, S. Raby, G. G. Ross, Phys. Lett. B131, 59 (1983); J. R. Ellis, D. V. Nanopoulos, M. Quiros, Phys. Lett. B174, 176 (1986).

[2] T. Banks, D. B. Kaplan, A. E. Nelson, Phys. Rev. D49, 779-787 (1994). hep-ph/9308292; B. de Carlos, J. A. Casas, F. Quevedo, E. Roulet, Phys. Lett. B318, 447-456 (1993). hep-ph/9308325].

[3] M. Endo, K. Hamaguchi, F. Takahashi, Phys. Rev. Lett. 96, 211301 (2006). hep-ph/0602061]; Phys. Rev. D74, 023531 (2006). hep-ph/0605091; S. Nakamura, M. Yamaguchi, Phys. Lett. 
B638, 389-395 (2006). hep-ph/0602081]; M. Dine, R. Kitano, A. Morisse, Y. Shirman, Phys. Rev. D73, 123518 (2006). hep-ph/0604140].

[4] K. Yamamoto, Phys. Lett. B168, 341 (1986).

[5] G. Lazarides, C. Panagiotakopoulos, Q. Shafi, Phys. Rev. Lett. 56, 557 (1986).

[6] D. H. Lyth, E. D. Stewart, Phys. Rev. Lett. 75, 201-204 (1995). hep-ph/9502417.

[7] D. H. Lyth, E. D. Stewart, Phys. Rev. D53, 1784-1798 (1996). hep-ph/9510204.

[8] T. Asaka, M. Kawasaki, Phys. Rev. D60, 123509 (1999). hep-ph/9905467.

[9] T. Asaka, J. Hashiba, M. Kawasaki, T. Yanagida, Phys. Rev. D58, 083509 (1998). hep-ph/9711501.

[10] G. F. Giudice, R. Rattazzi, Phys. Rept. 322, 419-499 (1999). hep-ph/9801271.

[11] A. de Gouvea, T. Moroi, H. Murayama, Phys. Rev. D56, 1281-1299 (1997). hep-ph/9701244.

[12] T. Banks, M. Dine, M. Graesser, Phys. Rev. D68, 075011 (2003). hep-ph/0210256.

[13] A. Vilenkin, E. P. S. Shellard, "Cosmic Strings and Other Topological Defects," Cambridge University Press, Cambridge, England (1994).

[14] Y. .B. Zeldovich, I. Y. .Kobzarev, L. B. Okun, Zh. Eksp. Teor. Fiz. 67, 3-11 (1974).

[15] A. Vilenkin, Phys. Rev. D23, 852-857 (1981).

[16] R. D. Peccei, H. R. Quinn, Phys. Rev. Lett. 38, 1440-1443 (1977).

[17] J. Preskill, S. P. Trivedi, F. Wilczek, M. B. Wise, Nucl. Phys. B363, 207-220 (1991).

[18] S. A. Abel, S. Sarkar, P. L. White, Nucl. Phys. B454, 663-684 (1995). hep-ph/9506359].

[19] See, for example, J. E. Kim, Phys. Rept. 150, 1-177 (1987).

[20] D. J. Gross, R. D. Pisarski, L. G. Yaffe, Rev. Mod. Phys. 53, 43 (1981).

[21] M. Ibe, R. Kitano, H. Murayama, Phys. Rev. D71, 075003 (2005). hep-ph/0412200.

[22] F. Riva, Phys. Lett. B690, 443-450 (2010). [arXiv:1004.1177 [hep-ph]].

[23] M. Kawasaki, K. Kohri, T. Moroi, Phys. Rev. D71, 083502 (2005). astro-ph/0408426;

M. Kawasaki, K. Kohri, T. Moroi, A. Yotsuyanagi, Phys. Rev. D78, 065011 (2008). arXiv:0804.3745 [hep-ph]].

[24] M. Gleiser, R. Roberts, Phys. Rev. Lett. 81, 5497-5500 (1998). astro-ph/9807260.

[25] T. Hiramatsu, M. Kawasaki, K. 'i. Saikawa, JCAP 1005, 032 (2010). arXiv:1002.1555 [astroph.CO]].

[26] M. Kawasaki, K. 'i. Saikawa, arXiv:1102.5628 [astro-ph.CO]].

[27] F. Takahashi, T. T. Yanagida, K. Yonekura, Phys. Lett. B664, 194-198 (2008). 
arXiv:0802.4335 [hep-ph]].

[28] M. Maggiore, Phys. Rept. 331, 283-367 (2000). gr-qc/9909001.

[29] V. M. Kaspi, J. H. Taylor, M. F. Ryba, Astrophys. J. 428, 713 (1994).

[30] A. N. Lommen, astro-ph/0208572.

[31] R. van Haasteren et al., arXiv:1103.0576 [astro-ph.CO]]; D. R. B. Yardley et al., [arXiv:1102.2230 [astro-ph.GA]].

[32] B. P. Abbott et al. [ LIGO Scientific and VIRGO Collaborations ], Nature 460, 990 (2009). arXiv:0910.5772 [astro-ph.CO]].

[33] M. Kramer, 2, astro-ph/0409020.

[34] https://www.advancedligo.mit.edu/

[35] K. Kuroda [ LCGT Collaboration ], Class. Quant. Grav. 27, 084004 (2010).

[36] N. J. Cornish, Phys. Rev. D65, 022004 (2002). gr-qc/0106058.

[37] B. S. Sathyaprakash, B. F. Schutz, Living Rev. Rel. 12, 2 (2009). [arXiv:0903.0338 [gr-qc]].

[38] N. Seto, S. Kawamura, T. Nakamura, Phys. Rev. Lett. 87, 221103 (2001). astro-ph/0108011.

[39] R. Easther, J. T. Giblin, E. A. Lim, W. I. Park and E. D. Stewart, JCAP 0805, 013 (2008) arXiv:0801.4197 [astro-ph]].

[40] I. Affleck, M. Dine, Nucl. Phys. B249, 361 (1985).

[41] E. D. Stewart, M. Kawasaki, T. Yanagida, Phys. Rev. D54, 6032-6039 (1996). hep-ph/9603324.

[42] D. -h. Jeong, K. Kadota, W. -I. Park, E. D. Stewart, JHEP 0411, 046 (2004). hep-ph/0406136.

[43] M. Kawasaki, K. Nakayama, Phys. Rev. D74, 123508 (2006). hep-ph/0608335].

[44] G. N. Felder, H. Kim, W. -I. Park, E. D. Stewart, JCAP 0706, 005 (2007). hep-ph/0703275.

[45] S. Kim, W. -I. Park, E. D. Stewart, JHEP 0901, 015 (2009). arXiv:0807.3607 [hep-ph]].

[46] L. Hui, E. D. Stewart, Phys. Rev. D60, 023518 (1999). hep-ph/9812345.

[47] M. Kusakabe, T. Kajino, T. Yoshida, G. J. Mathews, Phys. Rev. D80, 103501 (2009). arXiv:0906.3516 [hep-ph]].

[48] M. Kawasaki, M. Kusakabe, Phys. Rev. D83, 055011 (2011). [arXiv:1012.0435 [hep-ph]].

[49] J. Kang, M. A. Luty, S. Nasri, JHEP 0809, 086 (2008). hep-ph/0611322.

[50] M. Srednicki, K. A. Olive, J. Silk, Nucl. Phys. B279, 804 (1987).

[51] T. Asaka, M. Yamaguchi, Phys. Lett. B437, 51-61 (1998) hep-ph/9805449; Phys. Rev. D59, 
125003 (1999) hep-ph/9811451.

[52] E. J. Chun, D. Comelli, D. H. Lyth, Phys. Rev. D62, 095013 (2000). hep-ph/0008133.

[53] K. Choi, K. S. Jeong, W. -I. Park, C. S. Shin, JCAP 0911, 018 (2009). arXiv:0908.2154 [hep-ph]].

[54] W. -I. Park, JHEP 1007, 085 (2010). arXiv:1004.2326 [hep-ph]].

[55] K. Choi, E. J. Chun, H. D. Kim, W. I. Park, C. S. Shin, [arXiv:1102.2900 [hep-ph]].

[56] P. Sikivie, Phys. Rev. Lett. 48, 1156-1159 (1982).

[57] A. Vilenkin, A. E. Everett, Phys. Rev. Lett. 48, 1867-1870 (1982).

[58] T. Vachaspati, A. Vilenkin, Phys. Rev. D30, 2036 (1984).

[59] M. Nagasawa, M. Kawasaki, Phys. Rev. D50, 4821-4826 (1994). astro-ph/9402066.

[60] S. Chang, C. Hagmann, P. Sikivie, Phys. Rev. D59, 023505 (1999). hep-ph/9807374.

[61] T. Hiramatsu, M. Kawasaki, K. 'i. Saikawa, arXiv:1012.4558 [astro-ph.CO]].

[62] Y. Cui, S. P. Martin, D. E. Morrissey, J. D. Wells, Phys. Rev. D77, 043528 (2008). arXiv:0709.0950 [hep-ph]].

[63] M. Kawasaki, K. Miyamoto, K. Nakayama, arXiv:1105.4383 [hep-ph]]. 